mer Spuren von Tierepithelien hat, einen Nies- oder Asthmaanfall auslösen kann. Bei Allergien gegen Katzen genügen bereits Spuren an Katzenantigenen. Leider finden sie sich überall: in Schulen, im Kino und in anderen öffentlichen Gebäuden. Diesem Allergen kann man nicht entkommen.

Gebäude, in denen sich bestimmte Schimmelpilze ausgebreitet haben, sind ebenfalls für Allergiker ein großes Problem. Schimmelpilzallergien haben sich in skandinavischen Ländern in den letzten Jahren stark ausgebreitet, in denen durch eine bestimmte Architektur - beispielsweise begrünte Flachdächer - das Eindringen von Wasser begünstigt wurde und das gesamte Bauwerk mit Schimmel durchseucht wurde.

Die Bemühung, Allergene zu meiden, sollte in der Behandlung allergischer Erkrankungen an erster Stelle stehen. Medikamente lindern die Beschwerden und beugen vor, so lange sie eingenommen werden. In jedem Fall muß versucht werden, das Allergen durch den Allergologen exakt ermitteln zu lassen und sich dann über die Therapiemöglichkeit einer spezifischen Hyposensibilisierung $\mathrm{zu}$ infor- mieren. Mit den modernen Substanzen, die heute zur Hyposensibilisierung oder spezifischen Immuntherapie zur Verfügung stehen, werden Erfolgsraten bis $\mathrm{zu}$ 90\% mit langfristiger Wirkung nach Beendigung der dreijährigen Behandlung erreicht.

ÄDA-Pressekontakt, Bodelschwinghstr. 17, D-22337 Hamburg.

\title{
Allergien als Volkskrankheit: Zahlen und Fakten
}

A llergien auf dem Vormarsch. So lauten die Schlagzeilen, die in letzter Zeit immer häufiger zu lesen sind. Seit 1950 ist die Häufigkeit von Heuschnupfen und Asthma um bis zu $5 \%$ pro Jahr angestiegen. Ein weiterer Anstieg ist zu befürchten. Die aktuellen Ergebnisse der ISAAC-Studie (The International Study of Asthma and Allergies in Childhood) zeigen, daß Großbritannien der absolute Spitzenreiter in der Asthma-Statistik in Westeuropa ist. Aber Deutschland liegt schon an dritter Stelle.

Einer Umfrage zufolge glauben fast $40 \%$ der gesamten bundesdeutschen Bevölkerung, daß sie unter einer All-

\section{Jeder dritte Bundesbürger ist Allergiker}

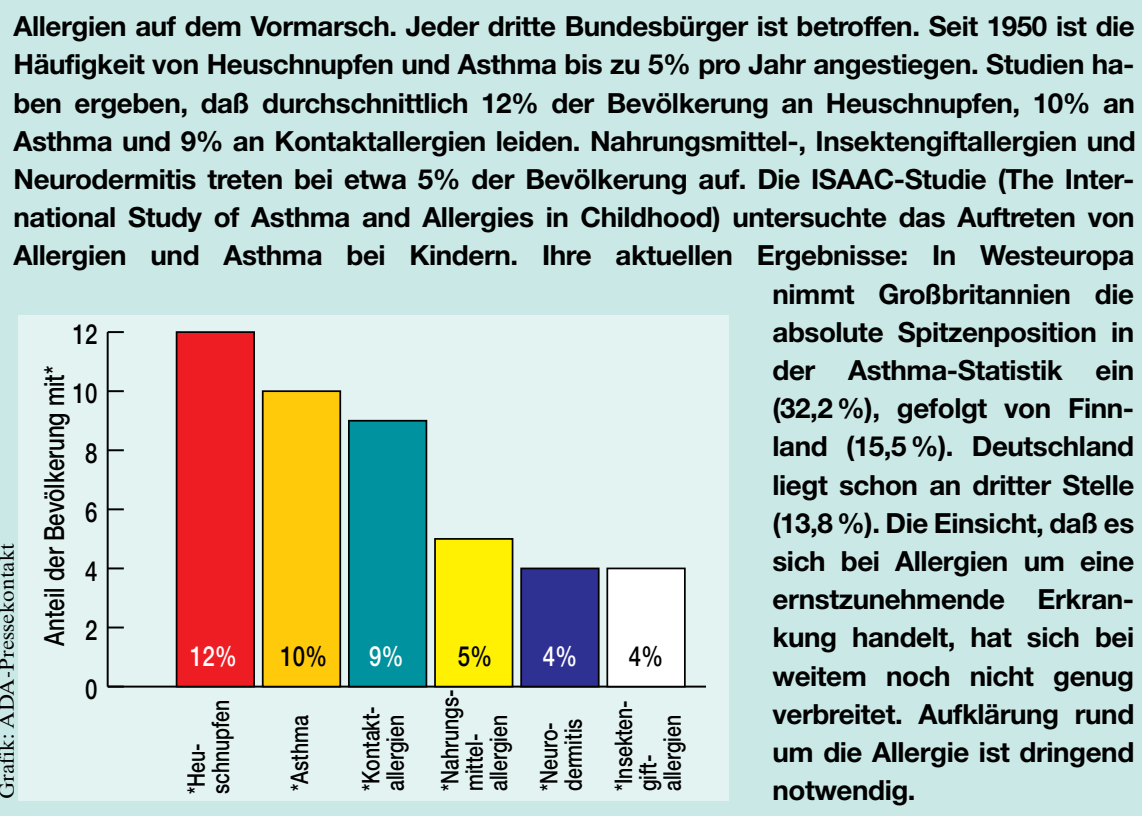

ergie leiden. Aber nicht alle diese Überempfindlichkeiten sind echte Allergien. So führte das Landesgesundheitsamt Brandenburg 1994 ein Allergievorsorge-Programm bei insgesamt 13700 ABC-Schützen in Berlin/Brandenburg durch. Die Resultate:

Bereits jedes vierte Schulkind litt an allergischen Erkrankungen. Und im höchsten Maße alarmierend ist dabei, daß fast jede zweite Allergie nicht bekannt war, allergische asthmatische Beschwerden sogar zu $70 \%$ nicht diagnostiziert worden waren.

Die Neigung, eine allergische Erkrankung zu entwickeln, ist erblich bedingt, doch spielen Zivilisationsfaktoren für ihre Entstehung eine entscheidende Rolle. Daß Allergien immer häufiger werden, ist ein alarmierendes Zeichen. Allergien werden häufig als lästige Bagatelle abgetan. Die Einsicht, daß es sich um eine ernstzunehmende Erkrankung handelt, hat sich bei weitem noch nicht genug verbreitet. Aufklärung rund um die Allergie ist dringend nötig.

Denn: Aus einem gewöhnlichen allergischen Schnupfen kann früher oder später eine schwere allergische Atemwegserkrankung werden: $43 \%$ der $\mathrm{Pa}$ tienten mit allergischem Schnupfen entwickeln innerhalb von 8 Jahren Asthma. Das statistische Bundesamt registrierte 1996 etwa 62000 asthmabedingte Krankenhauseinlieferungen. Damit es erst gar nicht $\mathrm{zu}$ diesem „Etagenwechsel“ kommt, ist der wichtigste Schritt eine Abklärung der Allergie und Nutzung der modernen Behandlungsmöglichkeiten $\mathrm{zu}$ einem frühen Zeitpunkt.

ÄDA-Pressekontakt, Bodelschwinghstr. 17, D-22337 Hamburg. 